Abstract S87 Table 1

\begin{tabular}{|c|c|c|c|c|c|c|c|c|}
\hline & $\underset{\text { Media }}{\text { A }}$ & $\begin{array}{c}\text { BL } \\
\text { Media }\end{array}$ & $\underset{\text { CSE }}{A}$ & $\begin{array}{c}\text { BL } \\
\text { CSE }\end{array}$ & $\begin{array}{c}\text { A } \\
\text { LPS }\end{array}$ & $\begin{array}{c}\text { BL } \\
\text { LPS }\end{array}$ & $\begin{array}{c}\text { A } \\
\text { CSE \& LPS }\end{array}$ & $\begin{array}{c}\text { BL } \\
\text { CSE \& LPS }\end{array}$ \\
\hline $\begin{array}{l}\mathrm{NS} \\
\text { IL-8 [pg/ml] }\end{array}$ & $24060 \pm 2514$ & $34490 \pm 3260$ & $36193 \pm 3755$ & $29417 \pm 2352$ & $34166 \pm 3347$ & $36969 \pm 5019$ & $43031 \pm 3211^{*}$ & $43890 \pm 5257$ \\
\hline $\begin{array}{l}\text { HS } \\
\text { IL-8 [pg/ml] }\end{array}$ & $28728 \pm 2422$ & $22903 \pm 3500$ & $41908 \pm 3269$ & $27995 \pm 4233$ & $37298 \pm 3052$ & $33298 \pm 5856$ & $39952 \pm 3131$ & $34095 \pm 5634$ \\
\hline $\begin{array}{l}\text { COPD } \\
\text { IL-8 [pg/ml] }\end{array}$ & $32163 \pm 2474$ & $43768 \pm 8730$ & $24376 \pm 2474^{*}$ & $21994 \pm 4176^{*}$ & $44092 \pm 3247$ & $54604 \pm 11363$ & $20866 \pm 1564^{*}$ & $27180 \pm 4680^{*}$ \\
\hline $\begin{array}{l}\mathrm{NS} \\
\text { IL-6 [pg/ml] }\end{array}$ & $227 \pm 32$ & $735 \pm 98$ & $253 \pm 35$ & $678 \pm 106$ & $391 \pm 53$ & $633 \pm 68$ & $469 \pm 48^{*}$ & $714 \pm 107$ \\
\hline $\begin{array}{l}\text { HS } \\
\text { IL-6 [pg/ml] }\end{array}$ & $289 \pm 48$ & $518 \pm 102$ & $285 \pm 53$ & $418 \pm 86$ & $447 \pm 77$ & $487 \pm 125$ & $536 \pm 55^{*}$ & $884 \pm 169 *$ \\
\hline $\begin{array}{l}\text { COPD } \\
\text { IL-6 [pg/ml] }\end{array}$ & $432 \pm 128$ & $846 \pm 235$ & $300 \pm 89$ & $728 \pm 113$ & $680 \pm 139$ & $1012 \pm 186$ & $324 \pm 43$ & $578 \pm 92$ \\
\hline
\end{tabular}

IL-8 and IL-6 release from well differentiated bronchial epithelial cell cultures into apical and basolateral compartments after stimulation with PA LPS \pm pre-treatment with $5 \%$ CSE for 24 h. NS: Non-smoker; HS: Healthy Smoker; A: Apical; BL: Basolateral.

Media: No stimulation; CSE: Stimulation with 5\% CSE 24 h; LPS: Stimulation with PA LPS 24 h; CSE \& LPS: Pre-treatment with $5 \%$ CSE 24 h, stimulation with PA LPS $24 \mathrm{~h}$.

* Statistically significant difference for soluble mediator release into a given compartment within each study group relative to the equivalent compartment without stimulation; $p<0.05$

phosphorylation patterns of oncoproteins in small clinical samples. Therefore we developed an exquisitely sensitive capillary is oelectric focusing immunoassay (cIEF) method for discriminating phosphorylated isoforms of ERK and AKT in lung tissue.

Methods Participants undergoing curative resection for early stage NSCLC were recruited. Samples from normal lung and tumour tissue were taken shortly after resection and analysed using cIEF.

Results Twenty patients were recruited (12 male, 8 female), mean age 67.3 years (SD 7.5), 10 current and 10 former smokers with a mean smoking exposure of 48.4 pack years (SD 24.4). Tumour tissue ( 9 squamous and 11 adenocarcinoma) was collected and paired macroscopically normal lung that was histologically normal $(n=7)$ or showed emphysema $(n=13)$, graded pathologically as mild $(n=7)$, moderate $(n=3)$ or severe $(n=3)$. The coefficient of variance was $7.6 \%$ for ERK and $4.2 \%$ for the AKT assay. The limits of detection and quantification were $8 \mathrm{ng}$ and $16 \mathrm{ng}$ of total protein respectively.

Diphosphorylated ERK (active form) was nearly three-fold higher in emphysematous lung tissue than normal lung tissue $(p=0.002)$ and was associated with pathological severity of emphysema. In contrast, diphosphorylated ERK did not differ between paired normal lung and tumour $(p=0.45)$ and there was no correlation of ERK status with age, gender, smoking status, tumour site, histology or stage. AKT was significantly more phosphorylated in tumour than matched normal lung. There was a trend for more phosphorylated AKT with poor differentiation but no correlation with tumour histology, size, site or stage of disease. Current smokers had more phosphorylated AKT than ex-smokers. There was no difference in the phosphorylation patterns of AKT according to age, gender or emphysema status.

Conclusions We found a strong relationship between diphosphorylated ERK and emphysema. By contrast, AKT phosphostatus was associated with lung cancer but not with emphysema. The requirement for just nanograms of protein defines the potential of this technique for determining the phosphostatus of oncogenic signalling proteins in tiny clinical samples.

\section{S89 EPITHELIAL MESENCHYMAL TRANSITION (EMT) IN CHRONIC OBSTRUCTIVE PULMONARY DISEASE (COPD) AIRWAYS IS ATTENUATED BY INHALED CORTICOSTEROIDS (ICS)}

doi:10.1136/thoraxjnl-2012-202678.095

'Sukhwinder Singh Sohal, 'Amir Soltani, ${ }^{2}$ David Reid, ${ }^{3}$ Chris Ward, 'Steven Weston, ${ }^{1}$ Hans Konrad Muller, 'Richard Wood-Baker, 'Eugene Haydn Walters. 'NHMRC Centre of Research Excellence in Chronic Respiratory Disease, School of Medicine, University of Tasmania, Hobart, Australia; 2Iron Metabolism Laboratory, Queensland Institute of Medical Research, Queensland, Australia. ${ }^{3}$ nnstitute of Cellular Medicine, Newcastle University, Newcastle Upon Tyne, United Kingdom

Introduction and Objectives We recently reported ${ }^{1,2}$ that EMT is active in the airways of COPD patients; in this process epithelial cells change shape and become motile, then digest through the reticular basement membrane ( $\mathrm{Rbm}$ ) which becomes fragmented and transition to a mesenchymal fibroblast-like cell. We also demonstrated that the Rbm is hyper-vascular, a combined picture specifically suggesting active EMT-type-III, which is a dangerous, pre-malignant condition. This may well be the link between COPD and lung cancer. In this study, we have assessed the effects of ICS on markers of EMT in endobronchial biopsies (ebb) in COPD.

Methods A double-blinded, randomised, placebo-controlled study assessed the effects of inhaled fluticasone propionate (FP: $500 \mu \mathrm{g}$ twice daily) on EMT in 34 COPD patients. Ebb were assessed for EMT related Rbm fragmentation (core structural marker) and immunostained for the EMT signatures S100A4 (a fibroblast epitope), matrix-metalloproteinase-9 (MMP-9) and the epithelial activation marker, epidermal growth factor receptor (EGFR).

Results Table 1.

Conclusions This is the first study to report the positive effects of ICS on EMT markers in COPD. This may be the mechanistic link between ICS and its reported preventive action against smokingrelated lung cancer in COPD.

\section{References}

1. Sohal SS, et al. Respirology 2010, 15(6):930-938.

2. Sohal SS, et al. Respir Res 2011, 12(1):130.

\section{Improving lung cancer survival}

\section{S90 THE NATIONAL LUNG CANCER AUDIT - NO EVIDENCE OF A "SEVEN-YEAR ITCH"}

doi:10.1136/thoraxjnl-2012-202678.096

'P Beckett, 'MD Peake, 'I Woolhouse, 'R Stanley. 'Royal College of Physicians, London, England; '2The Information Centre for health and social care, Leeds, England

Introduction The National Lung Cancer Audit, now in its $7^{\text {th }}$ year, is run jointly by the Royal College of Physicians and The Information Centre for health and social care, and is commissioned by the Healthcare Quality Improvement Partnership (HOIP). Its development was driven by the realisation that lung cancer outcomes vary widely across the UK and are poor compared to other western 